\title{
Gene therapy avenues and COVID-19 vaccines
}

\author{
Omar S. Abu Abed (1D)
}

Received: 18 January 2021 / Revised: 26 April 2021 / Accepted: 17 May 2021 / Published online: 2 June 2021

(c) The Author(s), under exclusive licence to Springer Nature Limited 2021

2020 has witnessed unprecedented situations due to coronavirus pandemic that affected all aspects of life. The whole globe lived months of uncertainty before two companies have announced the incredible results of phase III clinical trials for two different mRNA-based vaccines.

\section{To the Editor:}

BioNtech is a German start-up research and development company that has taken the initiative to develop an mRNA vaccine called (BNT162b2) in cooperation with a wellknown US-based pharmaceutical company, Pfizer. Similarly, Moderna is a juvenile company concerned with pioneering pharmaceutical biotechnological products, namely, RNA-based therapeutics, based in Cambridge, MA, and they recently announced their new vaccine, mRNA1273. Nine months of hard-working was enough to develop vaccines as a prophylactic step to protect people from being infected or highly affected after the infection. This may be one of the fastest drug development processes in history. It is not a coincidence that the first two vaccines that reached the terminal stages and have been approved by the FDA are utilising mRNA technology as a promising tool for the awaited vaccine. Both mRNAs are designed in a unique sequence to translate prefusion-stabilised conformation spike protein to stimulate the innate immune system to produce antibodies to be ready to invade the virus should infection happens. Both vaccines share the general idea and the main mRNA structure with some unique minor variations. The sequence of mRNA is 4100-4300 nucleotides long with a $5^{\prime}$ cap. The sequence also includes two proline substitutes (2P) that cause the spike to adopt a perfusionstabilised conformation to reduce membrane fusion and stimulate neutralising antibodies [1, 2]. Noteworthy, the mRNA does not include any uridine residues; however, they are replaced by 1-methyl-3'-pseudouridylyl [3].

Omar S. Abu Abed

Omar.abuabed@aaup.edu

1 Health Sciences Department, Arab American University in Palestine, Ramallah, Palestine
Typically, vaccines have one of the following forms: inactivated or live attenuated viruses, spike proteins or genetic materials (DNA or RNA) able to upregulate viral spike proteins when uptaken by the host's cells. While each approach has its pros and cons, mRNA-based vaccines possess unique facets not available in the other approaches. The main mechanism of mRNA vaccine is illustrated in Fig. 1. Firstly, mRNA-based vaccines manufacturing is easy and reproducible due to the well-developed and validated laboratory techniques used to obtain mRNA with specified and predefined characteristics. Moreover, delivering mRNA can avoid many complicated steps required to produce, express, and purify recombinant proteins in laboratories. Developing protein-based drugs or vaccines should pass several quality-control tests, stability assays, storage condition investigation and pharmacokinetic characterisation, while mRNA vaccines are utilising the natural machinery protein production inside human cells.

Furthermore, mRNA vaccines' production is a cell-free process and entirely in vitro with chemical processing without the need for advanced biological methods and cell culture techniques as many other viruses and protein-based vaccines need. Besides, mRNA has a very short half-life; this facilitates their elimination from the body after doing their work; thus, preventing any interaction with the packed human genetic materials [4]. In comparison to DNA vaccines, there is no need for mRNA to reach the nucleus of cells; it works efficiently once released inside the cytosol of cells. Therefore, DNA vaccines need external stimuli, e.g., electroporation, to facilitate DNA penetration and internalisation into the nucleus to incorporate with the host's cells. Finally, SAR-COV2 vaccines will be taken by billions of people from different races all over the world; this means we should look for the least allergic reaction inducing options. Although viral vectors are considered relatively safe, there are still some precautions and concerns around them, especially after 1999 when a 20-year-old man died 
Fig. 1 Illustration of the mechanism of action of mRNA vaccines and immune system response. Both mRNA vaccines are administered intramuscularly. Following the injection, lipid nanoparticles approach the cells and release mRNA inside the cytoplasmic space to encode a full-length mutated SARS-CoV-2 spike glycoprotein to be present at the out surface of the cells. The human immune system recognizes the spike protein and identifies it as a foreign body. Thus, antibodies are generated to fight the protein. Copies of the generated antibodies will be stored in the body to fight any potential COVID-19 infection in the future. Both vaccines are being given in two different jabs three to four weeks apart.

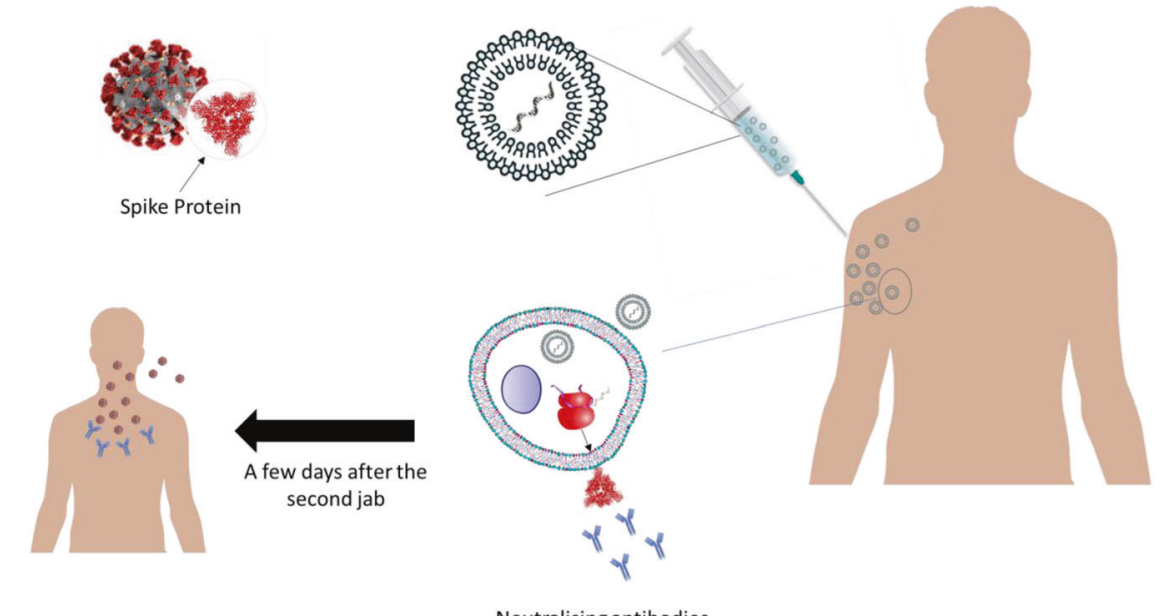

Neutralising antibodies due to an allergic reaction to the vehicle (inactive carrier) during a gene therapy clinical study in Pennsylvania [5]. Therefore, avoiding using viruses as carriers for vaccines or vaccines themselves may be a safer option; especially, with a wide range and a massive number of patients expecting to take the vaccine. Nevertheless, this type of reactions may happen with any carriers or any approach of vaccines.

Despite the above-mentioned advantages of mRNAbased vaccines, the delivery of mRNA, like many other biomacromolecules, is challenging and may not be attained without encasing them into vehicles. Pharmacokinetics, biodistribution, stability, cellular uptake, and targeting of naked mRNA after parenteral administration are crucial due to mRNA's short half-life [6]. Therefore, in BioNtechPfizer and Moderna vaccines, lipid nanoparticles are utilised to carry mRNA, protect them during the manufacturing and transportation processes, and enhance their stability in vivo, biodistribution and targeting. Nanocarriers are emergent promising drug delivery approaches that drew the attention of scientific communities in the previous decades. Several forms of nanocarriers and biomaterials were able to successfully deliver a wide range of drugs through various routes of administration [7-10]. Lipid nanoparticles are 100-200 ng miniaturised drug delivery systems, e.g., lipids micelles and liposomes. Fortunately, several liposomal products are FDA-approved to be marketed in different countries worldwide, such as AmBisome and Doxil ${ }^{\circ}$ for the delivery of Amphotericin B and Doxorubicin, respectively [11]. PEGylated liposomes are the used vehicles in both mRNA vaccines. Liposomes are nanocarriers comprised of a bilayer of phospholipids and cholesterol, which mimics animal cells' membrane and facilitate their fusion or penetration into cells to release their loaded mRNA. Many other materials have been used to deliver macromolecules, e.g., exosomes, polymers, and carbon derivatives. However, liposomes possess advantages over other nanocarriers for biodegradability, biocompatibility, cell membrane-like nature, ability to confine hydrophilic and hydrophobic drugs, and positive charge to maintain their encapsulation of negatively charged mRNA and consequently enhance their transfection into cells $[12,13]$. Once mRNA is free inside cells cytoplasm space, mRNA's translation process into SARS-COV2 spike protein stimulates innate immunity to produce neutralising antibodies against the produced protein.

Several hypotheses were suggested to explain the way of liposome-cells interaction and the factors affecting the interaction. The most popular interaction mechanisms are adsorption, endocytosis, lipid/protein exchange, and fusion [14]. Adsorption usually happens due to the charge difference, which is not the case here as the net charge of vaccine particles is not strong enough to form adsorption due to the different charge of mRNA and lipid into the same vesicle. Therefore, if adsorption does not lead to fusion or uptake, it will not be strong enough to form genetic materials exchange. Similarly, lipid or protein exchange probability is not high as it occurs when liposomes contain proteins that facilitate lipid exchange with lipoprotein included into the cell membrane [15]. Although the probability of adsorption is not high, adding PEG as a ligand to the membrane of liposomes facilitates the endocytosis and cellular uptake of the vaccine particles $[14,16]$. Fusion is facilitated by the inclusion of phosphatidylcholine, which is available in both vaccines in the form of DSPC; however, we do not expect that the main mechanism of unloading the gene is fusion since it is a very slow 
process and may take days which much longer than the half-life of mRNA [17]. In conclusion, we overweigh that the main mechanism of interaction with cells is endocytosis or so-called vesicular uptake.

Albeit the two vaccines utilise the same technology by upregulating the spike protein and stimulating the natural immunity against it, they are not identical. They do not possess the same potency, physicochemical characteristics, and storage conditions. One shot of Moderna's vaccine contains $100 \mu \mathrm{g}$ of mRNA, while $30 \mu \mathrm{g}$ of mRNA in the BioNtech-Pfizer vaccine is enough to protect and induce a prophylactic action as efficient as $100 \mu \mathrm{g}$ Moderna can do. The big difference in dose is questionable, and there is no definite explanation for it. The big difference in dose may be owed to the liposomal formulations or the nature of mRNA itself.

Changing the amount of lipids, the type of lipids, cholesterol: lipid ratio or more than one factor may significantly affect the transfection efficiency or the vaccine's pharmacokinetic properties, thus changing the required dose. Therefore, the inactive ingredients forming the vehicle may be the main reason behind the dose difference if other factors have no effects. Alternatively, the nature of mRNA, its exact sequence, purity, and sequencing method may play a crucial role in determining mRNA's potency. In our opinion, we overweight that mRNA has a major impact on determining the dose of the vaccine and made Pfizer-BioNtech vaccine more effective at smaller doses. The big difference in dose was not a result of changing the lipid ingredients. Noteworthy, the vaccine that contains $100 \mu \mathrm{g}$ should be stored at $-20^{\circ} \mathrm{C}$, while the vaccine with only $30 \mu \mathrm{g}$ mRNA should be strictly kept at $-70{ }^{\circ} \mathrm{C}$. None of the Pfizer-BioNtech vaccine lipid components needs to be stored at a temperature lower than $-20^{\circ} \mathrm{C}$; hence, the extremely cold temperature $\left(-70{ }^{\circ} \mathrm{C}\right)$ is required to stabilise mRNA rather than the liposomal formulation. As mRNA in BNT162b2 needs to be stored at ultra-cold temperature while mRNA in the counterpart vaccine remains stable for a very long time at the household freezers. It means tiny changes in the nucleic acid sequence led to the change in storage conditions and, accordingly, potency. Despite the sophistications accompanying Pfizer-BioNtech storage, it has, therefore, more advantages as the lower dose will enable the manufacturer to produce massive amounts of vaccine within a shorter time.

Having said that, both mRNA-based vaccines represent a unique case that is considered one of the most advanced and promising achievements in the field of pharmaceutical biotechnology and biopharmaceutical formulations. More than three decades of research effort on developing gene therapy solutions for many diseases could not convey many healthcare policymakers, pharmaceutical companies, funding agencies, medicine agencies, and drug administrations to adopt gene therapy avenues as highly potential approaches to transfer the therapeutic strategies into a new era. However, these mRNA vaccines, which have been developed and approved within a few months, signify a breakthrough in the field of gene therapy, which has battled to achieve ordinary acknowledgement due to a large number of sceptical and conservative scientists and other claimed safety and translational concerns. Although these two vaccines are not the first approved drugs utilising genetic materials as active ingredients, they are believed to be a milestone in modern medical history that may forever change pharmaceutical approaches.

Normally, viruses mutate frequently into several variants. However, the new coronavirus has lasted for a whole year without mutating into any mutations that significantly affected the virus's properties. Recently, several variants were detected; amongst them, three variants were the most popular, the UK, South Africa, and Brazil. These variants concern the whole scientific community and health care services due to significant changes in their spike gene. The variants' changes may affect the virus's external properties and affect its response to the developed vaccines. Therefore, developing vaccines that can adapt or work against a wide range of variants may overcome the challenges of spreading variants. Since alarming the risk of the variants is highly contagious and may be more dangerous than the original virus, scientists, pharmaceutical companies, governments, and drug regulators worldwide commenced the assessment of the existing vaccines against these variants.

A study conducted at Washington University School of Medicine suggests that the Pfizer-BioNtech vaccine is effective against the B.1.1.7 (UK) variant at the same dose we normally use. However, the other two variants need 3.5-10 folds of antibodies to be neutralised; this means the available dose is not highly effective against them, according to their study [18]. Another critical evaluation of the effectiveness of BNT162b2 against the variants suggests that the vaccine is effective against all variants except South African's one [19]. Similarly, the effect of the mRNA-1273 vaccine against the emerging variants is controversial. A correspondence published last February in the New England Journal of Medicine suggests that Moderna Vaccine is highly effective against the UK variants. However, the same study concludes that a significant reduction (2.3-6.4) in titers of neutralising antibodies against other variants was observed [20]. To overcome the risk associated with the South African variant, both Moderna and Pfizer-BioNtach have commenced developing booster shots of their vaccines to be given annually to the population to reduce the hospitalisation and death rate. Unlike other vaccines, mRNA vaccines are flexible and adaptable; they can be modified to suit different emergent variants in a very short time with 
minimum effort. The lipid carriers can be used for the coming vaccines with changes in the mRNA sequence only. mRNA sequencing is not a complicated process and can be performed within weeks, according to Ugur Shahin, the CEO and co-founder of BioNtech.

With the mass campaign of vaccine rollout worldwide, many countries and research centres carried out real-world studies on both vaccines. These studies aim to confirm, clarify and assure the provided results by the originators, and investigate further parameters, such as hospitalisation rate reduction and the protection of seniors. Also, these studies have faced a current of sceptical scientific communities and those who believe in the theory of conspiracy. The real-world studies are observational that can enable the science community to understand and watch the actual effect of the vaccine without any conflict of interest. An uncontrolled study was conducted in Israel on more than $500 \mathrm{~K}$ people who received the BNT162b2 mRNA vaccine compared to those who have not received any jab of the vaccine. The study suggests that the second dose of the vaccine protected more than $90 \%$ of people from either being infected or having asymptomatic COVID-19 than around $50 \%$ of those who received only one dose. Also, the hospitalisation rate and severe diseases raised by the infection were dropped after the second dose by $87 \%$ and $92 \%$, respectively. Death prevention is estimated to be $72 \%$ 2-3 weeks after the vaccine's first dose. The effectiveness of the vaccine in specific subpopulation in protecting the community from being infected or suffering from symptomatic disease was consistent across age groups [21]. Similarly, another real-world performed in the UK confirmed that the effectiveness of the Pfizer-BioNtech vaccine was $90 \%$ or more in elderly people with $>80$ years old. According to the same study, the first jab of the vaccine reduced the risk of hospital admission by $85 \%$ after 28 days. The effect of the Pfizer-BioNtech vaccine on mortality rate was also investigated, and it estimated that a single dose of the vaccine reduced the probability of death due to COVID-19 by $85 \%$ [22].

In addition to the BNT162b2 vaccine, the real-world studies also investigated the effectiveness of the Moderna vaccine. In the USA, a study was conducted to assess the protecting role of both mRNA vaccines, BNT162b2 and mRNA-1273, in the American community under conditions other than the randomised phase III clinical trials by the manufacturers. The obtained results are consistent with results provided by the vaccines' developers. The study demonstrated that both vaccines are highly protectant for people with high-risk factors of being infected of developing severe diseases as a result of COVID-19. These results were obtained comparing vaccinated individuals with at least one dose with their unvaccinated counterparts [23]. Interestingly, a non-peer-reviewed study was released on the 31st. of March 2021, demonstrating the safety and efficacy of BNT162b2 in children between 12 and 16 years old in the US. The study concludes that the vaccine is entirely safe and $100 \%$ effective in the adolescent subgroup; no infection was reported among those who received two jabs of vaccines compared to those who received placebo. Since children form a significant part of any community, they play a crucial role in disease transmission. Therefore, Pfizer and BioNtech will submit an Emergency Use Authorisation request to the FDA to approve the use of the vaccine in school pupils soon to facilitate and speed up the process of going back to normal [24].

This unprecedented achievement will also stress the crucial solutions that gene therapy may provide for many diseases. In the coming future, we expect to see a considerable effort for developing mRNA-based treatments for a wide range of diseases, e.g., hereditary disorders, type 1 Diabetes Mellitus, cancer, and HIV. Many other mRNA vaccines may also turn into reality for preventing infectious diseases and epidemics for being scalable, reproducible, versatile, and adaptable with different viruses' variants. mRNA vaccines provide flexibility to be modified if any new virus variants may appear; thus, producing new forms of the vaccine within a few weeks. This is a great opportunity for the FDA and EMA to revise the drug development pipeline to make it more flexible and less time-consuming.

\section{Compliance with ethical standards}

Conflict of interest The author declares no competing interests.

Publisher's note Springer Nature remains neutral with regard to jurisdictional claims in published maps and institutional affiliations.

\section{References}

1. Pallesen J, Wang N, Corbett KS, Wrapp D, Kirchdoerfer RN, Turner HL, et al. Immunogenicity and structures of a rationally designed prefusion MERS-CoV spike antigen. Proc Natl Acad Sci USA. 2017;114:E7348-E57.

2. Walsh EE, Frenck RW, Falsey AR, Kitchin N, Absalon J, Gurtman A, et al. Safety and immunogenicity of two RNA-based Covid-19 vaccine candidates. The New England J Med. 2020;383: 2439-50.

3. World Health Organization (September 2020). "Messenger RNA encoding the full-length SARS-CoV-2 spike glycoprotein". WHO MedNet.Archived from the original document on 5 January 2021. Retrieved 16 December 2020..

4. Sahin U, Kariko K, Tureci O. mRNA-based therapeuticsdeveloping a new class of drugs. Nat Rev Drug Discov. 2014; 13:759-80

5. Sibbald B. Death but one unintended consequence of genetherapy trial. CMAJ. 2001;164:1612.

6. Sergeeva OV, Koteliansky VE, Zatsepin TS. mRNA-based therapeutics-advances and perspectives. Biochemistry. 2016; 81:709-22. 
7. Abu Abed OS, Chaw CS, Williams L, Elkordy AA. PEGylated polymeric nanocapsules for oral delivery of trypsin targeted to the small intestines. Int J Pharmaceutics. 2021;592:120094.

8. Abu Abed OS, Chaw C, Williams L, Elkordy AA. Lysozyme and DNase I loaded poly (D, L lactide-co-caprolactone) nanocapsules as an oral delivery system. Sci Rep. 2018;8:13158.

9. Xu L, Faruqu FN, Liam-or R, Abu Abed O, Li D, Venner K, et al. Design of experiment (DoE)-driven in vitro and in vivo uptake studies of exosomes for pancreatic cancer delivery enabled by copper-free click chemistry-based labelling. J Extracell Vesicles. 2020;9:1779458.

10. Hong S-S, Oh KT, Choi H-G, Lim S-J. Liposomal formulations for nose-to-brain delivery: recent advances and future perspectives. Pharmaceutics. 2019;11:540.

11. Puri A, Loomis K, Smith B, Lee J-H, Yavlovich A, Heldman E, et al. Lipid-based nanoparticles as pharmaceutical drug carriers: from concepts to clinic. Crit Rev Ther Drug Carr Syst. 2009;26:523-80.

12. Elsana H, Olusanya TOB, Carr-Wilkinson J, Darby S, Faheem A, Elkordy AA. Evaluation of novel cationic gene based liposomes with cyclodextrin prepared by thin film hydration and microfluidic systems. Sci Rep. 2019;9:15120.

13. Olusanya TOB, Haj Ahmad RR, Ibegbu DM, Smith JR, Elkordy AA. Liposomal drug delivery systems and anticancer drugs. Molecules. 2018;23:907-24.

14. Kamps JAAM. Assessment of Liposome-Cell Interactions. In: Weissig V, editor. Liposomes: Methods and Protocols, Volume 2: Biological Membrane Models. Totowa, NJ: Humana Press; 2010. p. $199-207$.

15. Düzgüneş N, Nir S. Mechanisms and kinetics of liposome-cell interactions. Adv Drug Deliv Rev. 1999;40:3-18.
16. Miller CR, Bondurant B, McLean SD, McGovern KA, O’Brien DF. Liposome-cell interactions in vitro: effect of liposome surface charge on the binding and endocytosis of conventional and sterically stabilised liposomes. Biochemistry 1998;37:12875-83.

17. Yanagihara I, Kaneda Y, Inui K, Okada S. Liposome-mediated gene transfer. In: Dickson G, editor. Molecular and Cell Biology of Human Gene Therapeutics. Dordrecht. Netherlands: Springer; 1995. p. 64-82.

18. New evidence COVID-19 antibodies, vaccines less effective against variants [press release]. 2021.

19. Liu Y, Liu J, Xia H, Zhang X, Fontes-Garfias CR, Swanson KA, et al. Neutralising Activity of BNT162b2-Elicited Serum. 2021;1466-8.

20. Wu K, Werner AP, Koch M, Choi A, Narayanan E, Stewart-Jones GBE, et al. Serum neutralizing activity elicited by mRNA-1273 Vaccine. 2021;1468-70.

21. Dagan N, Barda N, Kepten E, Miron O, Perchik S, Katz MA, et al. BNT162b2 mRNA Covid-19 Vaccine in a Nationwide Mass Vaccination Setting. N Engl J Med. 2021;1412-23.

22. Bernal JL, Andrews N, Gower C, Stowe J, Robertson C, Tessier E, et al. Early effectiveness of COVID-19 vaccination with BNT162b2 mRNA vaccine and ChAdOx1 adenovirus vector vaccine on symptomatic disease, hospitalisations and mortality in older adults in England. 2021. https://doi.org/10.1101/2021.03.01. 21252652.

23. Pawlowski C, Lenehan P, Puranik A, Agarwal V, Venkatakrishnan A, Niesen MJM, et al. FDA-authorized COVID-19 vaccines are effective per real-world evidence synthesised across a multi-state health system. 2021. https://doi.org/10.1101/2021.02. 15.21251623 .

24. PFIZER-BIONTECH announce positive topline results of pivotal covid-19 vaccine study in adolescents [press release]. 2021. 